
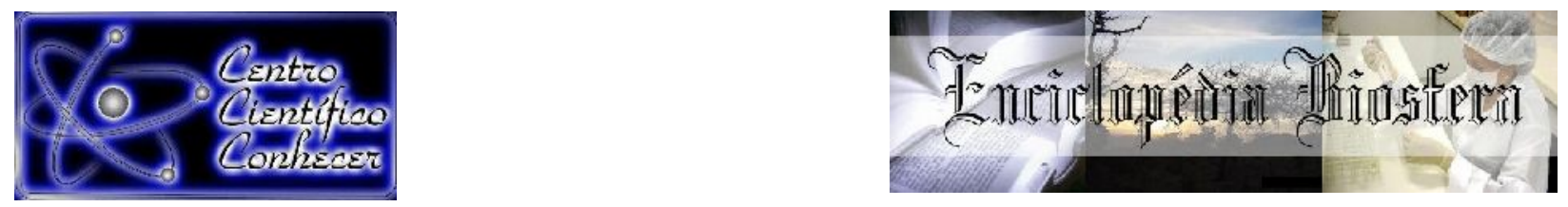

\title{
MÉTODOS ALTERNATIVOS AO USO DE ANIMAIS EM ENSINO E PESQUISA: EVOLUÇÃO E PANORAMA ATUAL DO BRASIL
}

\begin{abstract}
Desenir Adriano Pedro ${ }^{1}$
${ }^{1}$ Graduada em Medicina Veterinária, Mestranda no Programa de Pós-Graduação em Ciências e Biotecnologia (PPBI) e Bioterista do Núcleo de Pesquisa em Animais de Laboratório (NAL) - Universidade Federal Fluminense (UFF), Niterói, RJ, Brasil.

E-mail: desenirbio@gmail.com
\end{abstract}

Recebido em: 15/08/2021 - Aprovado em: 15/09/2021 - Publicado em: 30/09/2021

DOI: 10.18677/EnciBio_2021C28

trabalho licenciado sob licença Creative Commons Attribution-NonCommercial-NoDerivatives 4.0 International License.

\begin{abstract}
RESUMO
O uso de animais em atividades de ensino e pesquisa é pautado em princípios éticos que envolvem o conceito dos 3Rs - refine, reduce, replace - e em aspectos legais, que orientam e normatizam as atividades acadêmico-científicas com biomodelos. Com o passar das décadas, métodos alternativos à experimentação animal vêm sendo desenvolvidos, com o objetivo de substituir o uso de modelos animais ou reduzir e refinar sua utilização. O Conselho Nacional de Controle de Experimentação Animal - CONCEA dispõe sobre os critérios para o uso pedagógico e científico de animais no Brasil e, juntamente com a Rede Nacional de Métodos Alternativos - RENAMA e o Brazilian Center for the Validation of Alternative Methods - BraCVAM, realiza ações visando o desenvolvimento, a validação e o reconhecimento de metodologias alternativas. O País tem, atualmente, 25 métodos alternativos reconhecidos, que apresentam o total de nove desfechos. A partir de esforços intergovernamentais foi criada, em 2015, a Plataforma Regional de Métodos Alternativos ao Uso de Animais de Experimentação - PReMASUL, que objetiva a divulgação do tema nos países do MERCOSUL e a formação profissional. Em 2019 foi criado o primeiro curso de pós-graduação lato sensu em métodos alternativos ao uso de animais do Brasil, com o intuito de incentivar e ampliar a capacitação no país, a partir da especialização de profissionais na área. Embora o Brasil tenha uma legislação recente sobre experimentação animal, as pesquisas e ações em prol do desenvolvimento de métodos alternativos ao uso de animais têm avançado significativamente no país.
\end{abstract}

PALAVRAS-CHAVE: Animais de laboratório; Brasil; legislação; métodos alternativos. 


\title{
ALTERNATIVE METHODS TO THE USE OF ANIMALS IN TEACHING AND RESEARCH: EVOLUTION AND CURRENT OVERVIEW OF BRAZIL
}

\begin{abstract}
The use of animals in teaching and research activities is based on ethical principles that involve the concept of the 3Rs - refine, reduce, replace - and on legal aspects, which guide and standardize academic-scientific activities with biomodels. Over the decades, alternative methods to animal experimentation have been developed, with the objective of replacing the use of animal models or reducing and refining their use. The National Council for Control of Animal Experimentation - CONCEA provides for the criteria for the pedagogical and scientific use of animals in Brazil and, together with the National Network of Alternative Methods - RENAMA and the Brazilian Center for the Validation of Alternative Methods - BraCVAM, performs actions aimed at the development, validation, and recognition of alternative methodologies. The country currently has 25 recognized alternative methods, which present a total of nine outcomes. Based on intergovernmental efforts, in 2015 the Regional Platform for Alternative Methods for the Use of Experimental Animals - PReMASUL was created, which aims to promote the topic in MERCOSUR countries and professional training. In 2019, the first lato sensu postgraduate course on alternative methods to the use of animals was created in Brazil, with the aim of encouraging and expanding training in the country, based on the specialization of professionals in the area. Although Brazil has a recent legislation on animal experimentation, research, and actions in favor of the development of alternative methods to the use of animals have advanced significantly in the country.
\end{abstract}

KEYWORDS: Alternative methods; Brazil; laboratory animals; legislation.

\section{INTRODUÇÃO}

A utilização de animais para o desenvolvimento de análises in vivo é, ainda, etapa fundamental em muitos estudos científicos. Eles são empregados na investigação da resposta dos organismos vivos e de seus diferentes e complexos sistemas frente a estímulos conduzidos experimentalmente (BARRÉ-SINOUSSI; MONTAGUTELLI, 2015). Isso porque muitas pesquisas necessitam do uso de modelos animais para possibilitar o conhecimento de reações inesperadas que possam ocorrer a partir de intervenções terapêuticas, oriundas das interações fisiológicas, por exemplo (DI SANTO; APETREI, 2017).

O uso de biomodelos em atividades de ensino e pesquisa é pautado em preceitos éticos e legais. Em relação aos preceitos éticos, um dos pilares é o Princípio dos 3 Rs: refine, reduce e replace, proposto por Russel e Burch (1992). Neste princípio, eles abordam sobre o refinamento - refine - das técnicas empregadas, visando minimizar o sofrimento animal e as perdas desnecessárias; a redução - reduce - do número de animais, sendo indicada a utilização apenas da quantidade necessária para a obtenção de resultados confiáveis; e a substituição replace - dos animais por métodos alternativos, sempre que possível.

Relativo às questões legais, o Conselho Nacional de Controle de Experimentação Animal - CONCEA é o órgão com caráter normativo, deliberativo, consultivo e recursal, que coordena o uso pedagógico e experimental de animais no Brasil e é vinculado ao Ministério da Ciência, Tecnologia e Inovações - MCTI (BRASIL, 2009). O Conselho foi criado pela Lei Arouca, que estabelece critérios para o uso científico de animais no país (BRASIL, 2008). 
Antes da promulgação da legislação brasileira referente aos animais de laboratório, o seu uso para fins educacionais e científicos era citado no âmbito nacional apenas em lei que dispõe sobre sanções penais e administrativas derivadas de condutas prejudiciais e lesivas ao meio-ambiente, na qual é estabelecido que incorre nas penas de detenção e multa quem executa procedimento doloroso e cruel, ainda que com finalidade de ensino ou pesquisa, quando existirem recursos alternativos (BRASIL, 1998).

Nesse contexto, quando se trata de métodos alternativos ao uso de animais, o Brasil segue avançando no sentido de desenvolver e validar técnicas que possam substituir por completo a aplicação de animais em diversos testes ou reduzir e refinar a sua utilização. O CONCEA possui a Câmara Permanente de Métodos Alternativos ao Uso de Animais e, além desta Câmara, há no País a Rede Nacional de Métodos Alternativos - RENAMA e o Brazilian Center for the Validation of Alternative Methods - BraCVAM. Juntos, CONCEA, RENAMA e BraCVAM regulam e coordenam o desenvolvimento e a validação dos métodos alternativos no âmbito nacional (MORETTO; STEPHANO, 2019).

O empenho atual para o desenvolvimento e a divulgação de metodologias alternativas no Brasil parte de princípios que visam incentivar a perseverança na busca por alternativas para a substituição dos biomodelos; a veracidade em usar modelo animal vivo apenas quando não houver método alternativo que possibilite a sua substituição; e a responsabilidade em conhecer metodologias alternativas desenvolvidas em outras instituições para o ensino (CONCEA, 2016a).

\section{HISTÓRICO DOS MÉTODOS ALTERNATIVOS}

A conceituação e o desenvolvimento de métodos alternativos foram iniciados em 1969, no Reino Unido, com a criação do Fund for the Replacement of Animals in Medical Experiments - FRAME, sendo essa a primeira iniciativa em prol do Princípio dos $3 R$ s. Com o avanço da área científica e a descoberta de diferenças metabólicas entre humanos e animais, percebeu-se a necessidade do desenvolvimento de modelos alternativos validados, o que alavancou os estudos nessa área. A partir da criação do "Aninal Welfare Guideline", em 1986, a política de estímulo ao desenvolvimento de metodologias alternativas foi declarada (CONCEA, 2015).

Em 1989, foi instituído na Alemanha o Zentrealstelle zur ErfassungBewertung von Ersatz und Erganzungsmethoden zum Tierversuch - ZEBET, um centro de documentação e avaliação de métodos alternativos à experimentação animal, primeiro do mundo. Na sequência, centros para o desenvolvimento e coordenação da validação de ensaios alternativos foram formados em outros países. O European Centre for the Validation of Alternative Methods - ECVAM foi criado em 1991 pela comunidade europeia; o Interagency Coordinating Center for the Validation of Alternative Methods - ICCVAM foi estabelecido em 1997 pelas agências governamentais dos EUA; O Japanese Centre for the Validation of Alternative Methods - JaCVAM foi implantado no Japão em 2005; e, em 2013, o BraCVAM foi instituído oficialmente no Brasil (TRÉZ, 2018).

Como pilar para a validação dos métodos alternativos ao uso de animais, existe a Organization for Economic Cooperation and Development - OECD, uma organização intergovernamental, criada em 1959 e formada por 34 países da Europa, Pacífico e América do Norte. A OECD possui diretrizes de ensaios internacionalmente aceitos, utilizados por instituições independentes e organizações governamentais e industriais. As suas guidelines são publicadas em seções, sendo 
as relativas aos métodos alternativos ao uso de animais as da seção 4 , voltadas aos testes de produtos químicos (OECD, 2021).

O desenvolvimento de métodos validados pela OECD possibilitou a substituição do uso de animais em pesquisas de produtos cosméticos em muitos países. O teste de ingredientes de cosméticos do produto acabado, chamado de testing ban, assim como a comercialização de cosméticos acabados ou seus ingredientes que tenham sido testados em animais, o market ban, foram proibidos na União Europeia (UE) com a promulgação da sétima emenda da Diretriz de Cosméticos da UE em 2013 (CONCEA, 2015).

$\mathrm{Na}$ legislação brasileira não há normatização federal específica sobre a proibição do uso de animais em estudos de produtos cosméticos. Entretanto, alguns estados brasileiros têm regulamentações próprias nesse sentido (DISNER, 2019). São Paulo (2014) e Mato Grosso do Sul (2014) foram os primeiros a criar normas estaduais para proibir o teste de cosméticos ou de seus ingredientes em animais. Em seguida, Amazonas (2015), Paraná (2015) e Pará (2016) fizeram o mesmo. Depois foi a vez do Rio de Janeiro (2017), com a promulgação da Lei no 7.814 que proíbe testes de cosméticos e de seus ingredientes, assim como a venda de novos produtos cosméticos testados em animais. A lei carioca foi julgada constitucional pelo Supremo Tribunal Federal - STF em maio de 2021. Minas Gerais (2018) também vetou os testes de cosméticos a partir da Lei no 23.050. Santa Catarina (2020) e Espírito Santo (2021) foram os estados mais recentes a aderirem à proibição.

O Projeto de Lei nำ70, em tramitação, propõe a alteração dos artigos 14, 17 e 18 da Lei Arouca, para dispor sobre a vedação do uso de modelos animais em ensino, pesquisa e testes laboratoriais voltados ao desenvolvimento de cosméticos humanos e aumentar o valor da multa nos casos de violação de seus dispositivos (BRASIL, 2014). Além deste, há o Projeto de Lei $\mathrm{n}^{0} 1.031$, que visa a alteração do artigo $1^{\circ}$ da Lei Arouca para dispor sobre a proibição dos testes de cosméticos e de produtos de limpeza e de higiene pessoal em animais, além da obrigatoriedade da informação visível em embalagens de produtos comercializados dentro do território nacional, caso tenham sido testados em animais (BRASIL, 2021c).

Em 2012 foi publicado o "Guia para Avaliação de Segurança de Produtos Cosméticos", indicando metodologias de análise in silico, in vitro e testes validados pela OECD, dentre outras informações (ANVISA, 2012b). Entretanto, mesmo após o desenvolvimento e validação de outros métodos, o Guia não teve, ainda, edição atualizada.

No País, o CONCEA possui como uma de suas competências acompanhar e analisar a introdução de metodologias alternativas que substituam o uso de animais (BRASIL, 2008). A sua Câmara Permanente de Métodos Alternativos ao Uso de Animais, instituída desde a criação do Conselho em 2008, viabiliza o avanço dessas ações, através da instauração de diretrizes para a implantação de métodos validados no território brasileiro (MORETTO; STEPHANO, 2019).

Já a RENAMA, fundada em 2012, tem por objetivos: oferecer auxílio e treinamento técnico; monitorar os laboratórios associados; promover a excelência dos ensaios; incentivar a qualidade laboratorial e viabilizar o desenvolvimento, a validação e a certificação de novos métodos alternativos. A Rede é composta por laboratórios centrais, sendo estes o Instituto Nacional de Metrologia, Qualidade e Tecnologia - INMETRO, o Instituto Nacional de Controle de Qualidade em Saúde INCQS e o Laboratório Nacional de Biociências - LNBio, além de laboratórios associados. À sua instituição, foi definido o prazo de duração de cinco anos, a contar 
da publicação do ato normativo (BRASIL, 2012). Passados cinco anos, a RENAMA foi renovada por mais três anos (BRASIL, 2017). E, em 2021, houve mais uma renovação, igualmente com prazo de três anos (BRASIL, 2021a).

Em relação ao BraCVAM, o centro de validação brasileiro, este foi idealizado por Presgrave (2008) que, após ser promulgada a legislação brasileira de experimentação animal - a partir da Lei Arouca - verificou a necessidade da criação de um órgão direcionado à coordenação dos estudos relativos aos métodos alternativos no país. Em janeiro de 2012 foi assinado acordo de cooperação técnica entre a Fundação Oswaldo Cruz - FIOCRUZ e a Agência Nacional de Vigilância Sanitária - ANVISA, buscando viabilizar a criação do BraCVAM (ANVISA, 2012a). E em 2013, um ano após a criação da RENAMA, o BraCVAM foi finalmente instituído.

\section{CONCEITUAÇÃO DOS MÉTODOS ALTERNATIVOS AO USO DE ANIMAIS}

Os métodos alternativos ao uso de animais podem ser definidos como qualquer metodologia que possibilite substituir, reduzir ou refinar o uso pedagógico ou científico de biomodelos (CONCEA, 2015). Essas metodologias são definidas, ainda, como ensaios validados e aceitos internacionalmente que garantam resultados similares e com reprodutibilidade para alcançar, sempre que possível, a mesma finalidade dos procedimentos substituídos por métodos que permitam: não empregar modelos animais; usar espécies de ordem consideradas inferiores; utilizar número menor de animais; realizar ensaios com sistemas orgânicos ex vivo; ou refinar as metodologias para diminuir ou eliminar o desconforto animal (BRASIL, 2009).

No Brasil, as metodologias são classificadas pelo CONCEA como métodos alternativos validados e métodos alternativos reconhecidos. Os métodos alternativos validados são aqueles cuja confiabilidade e relevância para determinada finalidade foram estabelecidas por meio de diferentes etapas que envolvem o desenvolvimento da metodologia, sua pré-validação, validação propriamente dita e revisão por comitê de especialistas, sendo comprovada sua conformidade com ensaios realizados em centros de validação ou com estudos internacionais, podendo alcançar aceitação regulatória a nível internacional. Já os métodos alternativos reconhecidos são os ensaios alternativos validados que foram reconhecidos no país pelo CONCEA (CONCEA, 2014a).

Existe, ainda, a classificação de método alternativo válido, cuja técnica não necessariamente passou pelas etapas de validação, mas para a qual existe número suficiente de informações que demonstram sua relevância e confiabilidade (ANVISA, 2012b).

\section{ALTERNATIVAS AO USO DE ANIMAIS EM ENSINO E PESQUISA}

Em relação às atividades pedagógicas, o CONCEA (2021) determina que é proibido 0 uso de animais em atividades de ensino demonstrativas ou observacionais que não tenham como objetivo desenvolver habilidades e competências dos discentes envolvidos. Diante disso, o uso de animais deve ser integralmente substituído por outros recursos providos de conteúdo e qualidade suficientes para proporcionar condições de aprendizado, como vídeos e modelos computacionais. O uso de metodologias alternativas deve ser estimulado e induzido pelas Comissões de Ética no Uso de Animais - CEUAs e nos casos em que seja necessário o uso de animais em oposição a métodos alternativos, as justificativas devem ser claras. Sempre que possível, deve-se utilizar métodos alternativos para promover o preparo prévio dos alunos para as atividades didáticas envolvendo os 
animais e, semestralmente, os docentes devem esclarecer à CEUA institucional se não há disponíveis, ainda, alternativas para as atividades com animais (CONCEA, 2016c).

Existem muitas metodologias alternativas possíveis para a substituição do uso de animais no ensino. Silva et al. (2016) relatam sobre a possibilidade do uso de manequins, simuladores mecânicos, vídeos interativos e filmes, simuladores computacionais, análise anatômica ex vivo e estudos a campo e observacionais. De acordo com Furlan e Fisher (2020), o uso de cadáveres de animais é comum nas aulas de zoologia e em outros cursos. Os autores relatam, também, que o uso de modelos e softwares como alternativas ao animal permite ao discente simular o acesso, de maneira eficaz, a estruturas anatômicas que nem sempre são vistas em cadáveres animais, por desgastarem-se com facilidade pela manipulação frequente.

Conforme Scheid e Konflanz (2016), as tecnologias revolucionaram as atividades pedagógicas e científicas. As simulações computacionais viabilizam a confecção de ambientes virtuais que favorecem o aprendizado, além de servirem como recursos importantes em determinadas pesquisas. Saraiva et al. (2016) descrevem sobre o desenvolvimento de um software, chamado Pharmaware $\AA$, voltado ao ensino de farmacologia no curso de Medicina da Universidade Federal do Ceará - UFC. Após avaliação da metodologia, as atividades desenvolvidas por meio do software demonstraram-se igualmente eficazes à metodologia convencional com utilização de animal - em relação à consolidação de informações referentes às vias de administração de fármacos.

Em determinadas atividades práticas acadêmicas, principalmente as relacionadas aos cursos da área da Saúde, como a aferição da pressão arterial e a punção venosa, um método alternativo ao uso de animais comumente empregado é a execução do procedimento com os próprios acadêmicos servindo como modelo para seus colegas durante a aula. Contudo, assim como no uso de animais, o desenvolvimento de atividades didáticas utilizando seres humanos como modelos deve estar de acordo com o que rege os comitês de ética e as regulamentações (SCHEID; KONFLANZ, 2016).

Especificamente sobre atividades didáticas relacionadas à técnica cirúrgica, o CONCEA classifica como metodologia alternativa ao uso de modelos animais a substituição parcial ou total do uso de animais vivos. Na substituição parcial, devem ser utilizados cadáveres de animais no início da disciplina, com etapa posterior na qual pode-se utilizar animais vivos. Os cadáveres devem ser originários de pessoas físicas ou jurídicas, desde que não tenham sido eutanasiados para essa finalidade. Quanto aos animais vivos empregados na segunda etapa, estes podem ser, preferencialmente, os que necessitam ser submetidos a cirurgias de castração ou os que precisam passar pela rotina de clínica cirúrgica. Todas as técnicas executadas nos animais vivos devem ser supervisionadas por um médico veterinário, assim como os cuidados pós-operatórios, observada a aprovação do projeto pedagógico pela CEUA institucional. No caso da substituição total do uso de animais vivos, todo o treinamento no decorrer da disciplina será realizado em cadáveres (CONCEA, 2016b).

Referente às metodologias alternativas à experimentação animal, quando estiverem disponíveis métodos alternativos validados e reconhecidos no Brasil, os estudos toxicológicos devem ser conduzidos através da utilização destes, conforme norma vigente. Diante de ensaios para seleção inicial de substâncias, métodos in vitro devem ser considerados, caso não comprometam o objetivo do estudo (CONCEA, 2016c). 


\section{MÉTODOS ALTERNATIVOS VALIDADOS NO BRASIL}

Para a validação de métodos alternativos ao uso de animais em experimentação no Brasil, as instituições interessadas devem estar associadas à RENAMA. O CONCEA poderá reconhecer o ensaio alternativo validado por centros para validação, como o BraCVAM, ou por estudos colaborativos internacionais que tenham sido publicados em compêndios oficiais. O reconhecimento do método alternativo se dará por meio de deliberação plenária do Conselho, considerando o parecer da Câmara de Métodos Alternativos e ouvidos os órgãos oficiais apropriados. Após reconhecimento pelo CONCEA, fica estabelecido o prazo limite de cinco anos para a substituição obrigatória da metodologia original, que emprega animal, pelo método alternativo (CONCEA, 2014a). A ANVISA (2015) aceita os métodos alternativos reconhecidos pelo CONCEA.

Atualmente, o CONCEA reconhece 25 métodos alternativos ao uso de animais, que apresentam o total de nove desfechos. Dos 25 métodos, 17 estão previstos na Resolução Normativa (RN) no 18 (CONCEA, 2014b) e têm como objetivos as seguintes avaliações: potencial de irritação e corrosão da pele; potencial de irritação e corrosão ocular; potencial de fototoxicidade; absorção cutânea; potencial de sensibilização cutânea; toxidade aguda; e genotoxidade, conforme Quadro 1.

QUADRO 1 - Métodos alternativos reconhecidos pela Resolução Normativa no 18 do CONCEA e seus respectivos desfechos.

\begin{tabular}{|c|c|}
\hline MÉTODO ALTERNATIVO & DESFECHO \\
\hline $\begin{array}{l}\text { Método OECD TG } 430 \text { - Corrosão dérmica in vitro: Teste de } \\
\text { Resistência Elétrica Transcutânea }\end{array}$ & \multirow{4}{*}{$\begin{array}{l}\text { Avaliação do } \\
\text { potencial de irritação e } \\
\text { corrosão da pele }\end{array}$} \\
\hline $\begin{array}{l}\text { Método OECD TG } 431 \text { - Corrosão dérmica in vitro: Teste da } \\
\text { Epiderme Humana Reconstituída }\end{array}$ & \\
\hline $\begin{array}{l}\text { Método OECD TG } 435 \text { - Teste de Barreira de Membrana in } \\
\text { vitro }\end{array}$ & \\
\hline Método OECD TG 439 - Teste de irritação Cutânea in vitro & \\
\hline $\begin{array}{l}\text { Método OECD TG } 437 \text { - Teste de Permeabilidade e } \\
\text { Opacidade de Córnea Bovina }\end{array}$ & \multirow{3}{*}{$\begin{array}{c}\text { Avaliação do } \\
\text { potencial de irritação e } \\
\text { corrosão ocular }\end{array}$} \\
\hline Método OECD TG 438 - Teste de Olho Isolado de Galinha & \\
\hline $\begin{array}{l}\text { Método OECD TG } 460 \text { - Teste de Permeação de } \\
\text { Fluoresceína }\end{array}$ & \\
\hline $\begin{array}{l}\text { Método OECD TG } 432 \text { - Teste de Fototoxicidade in vitro } \\
\text { 3T3 NRU }\end{array}$ & $\begin{array}{l}\text { Avaliação do potencial de } \\
\text { fototoxicidade }\end{array}$ \\
\hline Método OECD TG 428 - Absorção Cutânea método in vitro & $\begin{array}{l}\text { Avaliação da } \\
\text { absorção cutânea }\end{array}$ \\
\hline $\begin{array}{l}\text { Método OECD TG } 429 \text { - Sensibilização Cutânea: Ensaio do } \\
\text { Linfonodo Local }\end{array}$ & \multirow{2}{*}{$\begin{array}{c}\text { Avaliação do } \\
\text { potencial de sensibilização } \\
\text { cutânea }\end{array}$} \\
\hline $\begin{array}{l}\text { Método OECD TG 442A e 442B - Versões não radioativas } \\
\text { do Ensaio do Linfonodo Local }\end{array}$ & \\
\hline $\begin{array}{l}\text { Método OECD TG } 420 \text { - Toxicidade Aguda Oral - } \\
\text { Procedimento de Doses Fixas }\end{array}$ & \multirow{4}{*}{$\begin{array}{l}\text { Avaliação de toxicidade } \\
\text { aguda }\end{array}$} \\
\hline $\begin{array}{l}\text { Método OECD TG } 423 \text { - Toxicidade Aguda Oral - Classe } \\
\text { Tóxica Aguda }\end{array}$ & \\
\hline $\begin{array}{l}\text { Método OECD TG } 425 \text { - Toxicidade Aguda Oral - } \\
\text { procedimento "Up and Down" }\end{array}$ & \\
\hline $\begin{array}{l}\text { Método OECD TG } 129 \text { - estimativa da dose inicial para } \\
\text { teste de toxicidade aguda oral sistêmica }\end{array}$ & \\
\hline $\begin{array}{l}\text { Método OECD TG } 487 \text { - Teste do Micronúcleo em Célula } \\
\text { de Mamífero in vitro }\end{array}$ & $\begin{array}{l}\text { Avaliação de } \\
\text { genotoxicidade }\end{array}$ \\
\hline ntro Científico Conhe & \\
\hline
\end{tabular}


Outros sete métodos alternativos foram reconhecidos a partir da publicação da RN no 31 (CONCEA, 2016d) e apresentam como desfechos as avaliações do potencial de irritação e corrosão ocular; do potencial de sensibilização cutânea; da toxicidade reprodutiva; e da contaminação pirogênica em produtos injetáveis (Quadro 2).

QUADRO 2 - Métodos alternativos reconhecidos pela Resolução Normativa № 31 do CONCEA e seus respectivos desfechos.

\begin{tabular}{|l|c|}
\hline \multicolumn{1}{|c|}{ MÉTODO ALTERNATIVO } & DESFECHO \\
\hline $\begin{array}{l}\text { Método OECD TG 491 - Teste in vitro de curta duração } \\
\text { para danos oculares }\end{array}$ & $\begin{array}{c}\text { Avaliação do } \\
\text { potencial de irritação e } \\
\text { corrosão ocular }\end{array}$ \\
\cline { 1 - 1 } $\begin{array}{l}\text { Método OECD TG 492 - Epitélio corneal humano } \\
\text { reconstituído }\end{array}$ & $\begin{array}{c}\text { Avaliação do } \\
\text { pétodo OECD TG 442C - Sensibilização cutânea insibilização } \\
\text { cutânea } \\
\text { chemico }\end{array}$ \\
\cline { 1 - 1 } Método OECD TG 442D - Sensibilização cutânea in vitro & $\begin{array}{c}\text { Avaliação de toxicidade } \\
\text { reprodutiva }\end{array}$ \\
\hline $\begin{array}{l}\text { Método OECD TG 421 - Teste de triagem para toxicidade } \\
\text { reprodutiva e do desenvolvimento }\end{array}$ \\
$\begin{array}{l}\text { Método OECD TG 422 - Estudo de toxicidade repetida } \\
\text { combinado com teste de toxicidade reprodutiva }\end{array}$ & $\begin{array}{c}\text { Avaliação da contaminação } \\
\text { pirogênica em produtos } \\
\text { injetáveis }\end{array}$ \\
\hline Teste de Endotoxina Bacteriana (Farmacopeia Brasileira) & \\
\hline
\end{tabular}

O último método reconhecido, até o momento, foi o descrito na $\mathrm{RN}$ no 45 (CONCEA, 2019), o Teste de Ativação de Monócitos, cujo desfecho é a avaliação da contaminação pirogênica em produtos injetáveis (Quadro 3).

QUADRO 3 - Método alternativo reconhecido pela Resolução Normativa ํo 45 do CONCEA e seu desfecho.

\begin{tabular}{|c|c|}
\hline MÉTODO ALTERNATIVO & DESFECHO \\
\hline Teste de Ativação de Monócitos & $\begin{array}{c}\text { Avaliação da contaminação } \\
\text { pirogênica em produtos } \\
\text { injetáveis }\end{array}$ \\
\hline
\end{tabular}

Os métodos para avaliação do potencial de irritação e corrosão dérmica utilizam técnicas in vitro, com o emprego de amostras de pele animal, de pele humana reconstituída ou de membrana artificial similar à pele. A avaliação do potencial de irritação e corrosão ocular aplicam nas análises córnea bovina, córnea de galinha, córnea de coelho, rim de cão e modelos tridimensionais comerciais produzidos a partir de queratinócitos humanos. O potencial de fototoxicidade é analisado por meio de linhagem celular de fibroblastos murinos. A absorção dérmica é testada com amostra de pele. O potencial de sensibilização dérmica emprega análise in vivo em camundongo, porém com quantidade reduzida de animais, além de linhagem celular derivada de queratinócitos humanos para ensaio in vitro e método abiótico por ensaio in chemico (DISNER, 2019).

$\mathrm{Na}$ avaliação da toxidade aguda, evita-se usar a morte dos animais como marcador final da análise, baseando-se na observação de sinais evidentes de toxicidade, além de ser utilizado número reduzido de animais. O ensaio de genotoxicidade aplica em sua metodologia linfócitos de sangue periférico humano e de outros mamíferos, assim como linhagens celulares de roedores. Nas pesquisas ENCICLOPÉDIA BIOSFERA, Centro Científico Conhecer - Jandaia-GO, v. 18 n. 37 ; p. 341 2021 
de toxidade reprodutiva é realizado teste de triagem para avaliar parâmetros reprodutivos e, também, a análise de doses repetidas por tempo limitado. Já na avaliação da contaminação pirogênica em produtos injetáveis, é usada endotoxina bacteriana (DISNER, 2019) e técnica utilizando linhagem celular monocitária (SILVA et al., 2018).

\section{CAPACITAÇÃO EM MÉTODOS ALTERNATIVOS AO USO DE ANIMAIS}

O Brasil lidera a Plataforma Regional de Métodos Alternativos ao Uso de Animais de Experimentação - PReMASUL, criada em 2015 pelo MCTI. A plataforma tem como objetivos oferecer cursos para disseminação do tema "métodos alternativos" nos países do MERCOSUL, promover a criação de infraestrutura laboratorial e formar profissionais especializados, entrando em consonância com o panorama internacional que fomenta o Princípio dos 3Rs (BRASIL, 2021b).

Além de promover a criação da PReMASUL, o Brasil foi pioneiro ao estabelecer a pós-graduação lato sensu em métodos alternativos, primeiro curso de especialização do país na área, oferecido pelo Instituto de Ciência e Tecnologia em Biomodelos - ICTB da FIOCRUZ desde 2019. A pós-graduação tem como premissa oferecer formação básica consolidada, focada nos conceitos de bioética, experimentação animal, métodos alternativos e validação, de modo relacionado e aplicado (ICTB, 2021).

\section{CONSIDERAÇÕES FINAIS}

Diante do exposto é possível concluir que, mesmo sendo recente a promulgação da legislação acerca dos animais de laboratório no Brasil, a busca pelo desenvolvimento e validação de métodos alternativos ao uso de animais em ensino e pesquisa têm evoluído significativamente nos últimos anos. Diferentes órgãos atuam conjuntamente, visando a condução de pesquisas e a regulamentação de metodologias que possam substituir, reduzir ou refinar o uso de animais em atividades científicas. Ações integradas, voltadas ao incentivo e à promoção dos métodos alternativos e que fomentam a pesquisa e a capacitação de profissionais na área, contribuem para o progresso da temática no país e favorecem a projeção do Brasil no panorama internacional do desenvolvimento de alternativas ao uso de biomodelos animais.

\section{AGRADECIMENTOS}

A todos os pesquisadores que, em sua trajetória acadêmico-profissional, dedicam-se ao desenvolvimento de métodos alternativos ao uso de animais.

\section{REFERÊNCIAS}

AMAZONAS. Lei № 289, de 3 de dezembro de 2015. Proíbe a utilização de animais para desenvolvimento, experimentos e testes de produtos cosméticos, de higiene pessoal, perfumes e seus componentes no Estado do Amazonas, e dá outras providências. Diário Oficial do Estado do Amazonas, 2015. Disponível em: <https://sapl.al.am.leg.br/norma/9412>.

ANVISA - Agência Nacional de Vigilância Sanitária. Extrato de acordo de cooperação técnica, de 17 de janeiro de 2012. Diário Oficial da União de 18 de janeiro de 2012, Seção 3, p.122, 2012a. Disponível em: <https://www.incqs.fiocruz.br/images/stories/incqs/bracvam/Termo_BraCVAM_DOU. PDF>. 
ANVISA - Agência Nacional de Vigilância Sanitária. Guia para avaliação de segurança de produtos cosméticos. 2. Ed. Brasília, 2012b. Disponível em: $<$ https://www.gov.br/anvisa/pt-

br/centraisdeconteudo/publicacoes/cosmeticos/manuais-e-guias/guia-paraavaliacao-de-seguranca-de-produtos-cosmeticos.pdf/view $>$.

ANVISA - Agência Nacional de Vigilância Sanitária. Resolução - RDC № 35, de 7 de agosto de 2015. Dispõe sobre a aceitação dos métodos alternativos de experimentação animal reconhecidos pelo Conselho Nacional de Controle de Experimentação Animal - CONCEA. Diário Oficial da União de 10 de agosto de 2015, Seção 1, p.44, 2015. Disponível em: <https://www.in.gov.br/materia//asset_publisher/Kujrw0TZC2Mb/content/id/32389206/do1-2015-08-10-resolucaordc-n-35-de-7-de-agosto-de-2015-32389026>.

BARRÉ-SINOUSSI, F.; MONTAGUTELLI, X. Animal models are essential to biological research: issues and perspectives. Future Science AO, v. 1, n. 4, FSO63, 2015. Disponível em: <https://www.ncbi.nlm.nih.gov/pmc/articles/PMC5137861/>. doi: $10.4155 /$ fso.15.63.

BRASIL - Ministério da Ciência, Tecnologia e Inovação. Portaria № 491, de 03 de julho de 2012. Institui a Rede Nacional de Métodos Alternativos - RENAMA e sua estrutura no âmbito do Ministério da Ciência, Tecnologia e Inovação - MCTI, que será supervisionada por um Conselho Diretor. Diário Oficial da União de 05 de julho de 2012, Seção 1, Pág. 19, 2012. Disponível em: <https://antigo.mctic.gov.br/mctic/opencms/legislacao/portarias/migracao/Portaria_M CTI_n_491_de_03072012.html>.

BRASIL - Ministério da Ciência, Tecnologia e Inovação. Portaria № 4.556, de 15 de março de 2021. Cria a Rede Nacional de Métodos Alternativos ao Uso de Animais RENAMA no âmbito do Ministério da Ciência, Tecnologia e Inovações. Diário Oficial da União de 17 de março de 2021, Seção 1, Pág. 4, 2021a. Disponível em: <https://www.in.gov.br/web/dou/-/portaria-gm-n-4.556-de-15-de-marco-de-2021309000224>.

BRASIL - Ministério da Ciência, Tecnologia e Inovação. Portaria № 3.586, de 30 de junho de 2017. Renova a Rede Nacional de Métodos Alternativos - RENAMA e dá outras providências. Diário Oficial da União de 6 de julho de 2017, Seção 1, Pág. 8, 2017. $<$ https://antigo.mctic.gov.br/mctic/export/sites/institucional/ciencia/SEPED/Saude/arq uivos/PORTARIA-No-3.586-DE-30-DE-JUNHO-DE-2017.pdf>.

BRASIL - Ministério da Ciência, Tecnologia e Inovação. PReMASUL. Consulta institucional, 2021b. Disponível em: <https://antigo.mctic.gov.br/mctic/opencms/ciencia/SEPED/Saude/PReMASUL/PRe MASUL.html>.

BRASIL. Decreto № 6.899, de 15 de julho de 2009. Dispõe sobre a composição do Conselho Nacional de Controle de Experimentação Animal - CONCEA, estabelece as normas para o seu funcionamento e de sua Secretaria-Executiva, cria o Cadastro das Instituições de Uso Científico de Animais - CIUCA, mediante a regulamentação 
da Lei $n^{0} 11.794$, de 8 de outubro de 2008, que dispõe sobre procedimentos para o uso científico de animais, e dá outras providências. Diário Oficial da União de 16 de julho de 2009, Seção 1, Pág. 2, 2009. Disponível em: <http://www.planalto.gov.br/ccivil_03/_ato2007-2010/2009/decreto/d6899.htm>.

BRASIL. Lei № 11.794, de 8 de outubro de 2008. Regulamenta o inciso VII do parágrafo $1^{\circ}$ do artigo 225 da Constituição Federal, estabelecendo procedimentos para o uso científico de animais; revoga a Lei oㅡ 6.638, de 8 de maio de 1979; e dá outras providências. Diário Oficial da União de 9 de outubro de 2008, Seção 1, Pág. 8, 2008. Disponível em: <http://www.planalto.gov.br/ccivil_03/_ato20072010/2008/lei/l11794.htm>.

BRASIL. Lei № 9.605, de 12 de fevereiro de 1998. Dispõe sobre as sanções penais e administrativas derivadas de condutas e atividades lesivas ao meio ambiente, e dá outras providências. Diário Oficial da União de 13 de fevereiro de 1998, Seção 1, Pág. 1, 1998. Disponível em: <https://www.planalto.gov.br/ccivil_03/leis/l9605.htm>.

BRASIL. Projeto de Lei da Câmara № 70, de 2014. Altera dispositivos dos arts. 14, 17 e 18 da Lei no 11.794, de 8 de outubro de 2008, para dispor sobre a vedação da utilização de animais em atividades de ensino, pesquisas e testes laboratoriais com substâncias para o desenvolvimento de produtos de uso cosmético em humanos e aumentar os valores de multa nos casos de violação de seus dispositivos. Diário do Senado Federal de 16 de julho de 2014, Págs. 216-218, 2014. Disponível em: $<$ https://legis.senado.leg.br/diarios/ver/19013?sequencia=216>.

BRASIL. Projeto de Lei № 1.031, de 2021. Altera a Lei o 11.794, de 8 de outubro de 2008, para proibir os testes de cosméticos em animais e dá outras providências. Câmara dos Deputados, 2021c. Disponível em: $<$ https://www.camara.leg.br/proposicoesWeb/fichadetramitacao?idProposicao=22751 $31>$.

CONCEA - Conselho Nacional de Controle de Experimentação Animal. Resolução Normativa № 25, de 29 de setembro de 2015. Baixa o Capítulo "Introdução Geral" do Guia Brasileiro de Produção, Manutenção ou Utilização de Animais para Atividades de Ensino ou Pesquisa Científica do Conselho Nacional de Controle e Experimentação Animal - CONCEA. Diário Oficial da União de 2 de outubro de 2015, Seção 1, Pág. 4, 2015. Disponível em: <https://antigo.mctic.gov.br/mctic/export/sites/institucional/institucional/concea/arquiv os/legislacao/resolucoes_normativas/Resolucao-Normativa-CONCEA-n-25-de29.09.2015-D.O.U.-de-02.10.2015-Secao-I-Pag.-04_ANEXO-retificado-no-DOU-de06-10-2015.pdf>.

CONCEA - Conselho Nacional de Controle de Experimentação Animal. Resolução Normativa № 32, de 6 de setembro de 2016. Baixa as Diretrizes de Integridade e de Boas Práticas para Produção, Manutenção ou Utilização de Animais em Atividades de Ensino ou Pesquisa Científica. Diário Oficial da União de 8 de setembro de 2016, Seção 1, Pág. 05, 2016a. Disponível em: $<$ https://antigo.mctic.gov.br/mctic/export/sites/institucional/institucional/concea/arquiv os/legislacao/resolucoes_normativas/Resolucao-Normativa-CONCEA-n-32-de06.09.2016-D.O.U.-de-08.09.2016-Secao-I-Pag.-05.pdf>. 
CONCEA - Conselho Nacional de Controle de Experimentação Animal. Resolução Normativa № 45, de 22 de outubro de 2019. Reconhece método alternativo ao uso de animais em atividades de pesquisa no Brasil. Diário Oficial da União de 25 de outubro de 2019, Seção 1, Pág. 14, 2019. Disponível em: $<$ https://antigo.mctic.gov.br/mctic/export/sites/institucional/institucional/concea/arquiv os/legislacao/resolucoes_normativas/Resolucao-Normativa-n-45.pdf>.

CONCEA - Conselho Nacional de Controle de Experimentação Animal. Orientação Técnica № 9, de 18 de agosto de 2016. Orienta sobre alternativas ao uso de animais em disciplina de técnica cirúrgica. Diário Oficial da União de 19 de agosto de 2016, Seção 1, Pág. 4, 2016b. Disponível em: $<$ https://antigo.mctic.gov.br/mctic/export/sites/institucional/institucional/concea/arquiv os/legislacao/orientacoes_tecnicas/ORIENTACAO-TECNICA-N.-9-DE-18-DEAGOSTO-DE-2016.pdf>.

CONCEA - Conselho Nacional de Controle de Experimentação Animal. Resolução Normativa № 17, de 3 de julho de 2014. Dispõe sobre o reconhecimento de métodos alternativos ao uso de animais em atividades de pesquisa no Brasil e dá outras providências. Diário Oficial da União de 4 de julho de 2014, Seção 1, Pág. 51, 2014a. Disponível em: $<$ https://antigo.mctic.gov.br/mctic/export/sites/institucional/institucional/concea/arquiv os/legislacao/resolucoes_normativas/Resolucao-Normativa-CONCEA-n-17-de03.07.2014-D.O.U.-de-04.07.2014-Secao-I-Pag.-51.pdf>.

CONCEA - Conselho Nacional de Controle de Experimentação Animal. Resolução Normativa № 18, de 24 de setembro de 2014. Reconhece métodos alternativos ao uso de animais em atividades de pesquisa no Brasil, nos termos da Resolução Normativa $n^{\circ}$ 17, de 03 de julho de 2014, e dá outras providências. Diário Oficial da União de 25 de setembro de 2014, Seção 1, Pág. 9, 2014b. Disponível em: <https://antigo.mctic.gov.br/mctic/export/sites/institucional/institucional/concea/arquiv os/legislacao/resolucoes_normativas/Resolucao-Normativa-CONCEA-n-18-de24.09.2014-D.O.U.-de-25.09.2014-Secao-I-Pag.-9.pdf>.

CONCEA - Conselho Nacional de Controle de Experimentação Animal. Resolução Normativa № 30, de 2 de fevereiro de 2016. Baixa a Diretriz Brasileira para o Cuidado e a Utilização de Animais em Atividades de Ensino ou de Pesquisa Científica - DBCA. Diário Oficial da União de 03 de fevereiro de 2016, Seção 1, Pág. 3, 2016c. Disponível em: <https://antigo.mctic.gov.br/mctic/export/sites/institucional/institucional/concea/arquiv os/legislacao/resolucoes_normativas/Resolucao-Normativa-CONCEA-n-30-de02.02.2016-D.O.U.-de-03.02.2016-Secao-I-Pag.-03.pdf>.

CONCEA - Conselho Nacional de Controle de Experimentação Animal. Resolução Normativa № 31, de 18 de agosto de 2016. Reconhece métodos alternativos ao uso de animais em atividades de pesquisa no Brasil. Diário Oficial da União de 19 de agosto de 2016, Seção 1, Pág. 4, 2016d. Disponível em: $<$ https://antigo.mctic.gov.br/mctic/export/sites/institucional/institucional/concea/arquiv os/legislacao/resolucoes_normativas/Resolucao-Normativa-CONCEA-n-31-de18.08.2016-D.O.U.-de-19.08.2016-Secao-I-Pag.-04.pdf>. 
CONCEA - Conselho Nacional de Controle de Experimentação Animal. Resolução Normativa № 53, de 19 de maio de 2021. Dispõe sobre restrições ao uso de animais em ensino, em complemento à Diretriz Brasileira para o Cuidado e a Utilização de Animais em Atividades de Ensino ou de Pesquisa Científica - DBCA. Diário Oficial da União de 24 de maio de 2021, Seção 1, Pág. 10, 2021. Disponível em: <https://www.in.gov.br/web/dou/-/resolucao-normativa-n-53-de-19-de-maio-de2021-321569251>.

DI SANTO, J.P.; APETREI, C. Animal models for viral diseases: non-human primate and humanized mouse models for viral infections. Current Opinion in Virology, v. 25, p. v-vii, $2017 . \quad$ Disponível em: <https://www.sciencedirect.com/science/article/abs/pii/S1879625717301335?via\%3D ihub>. doi: 10.1016/j.coviro.2017.09.001.

DISNER, G.R. Métodos alternativos à experimentação animal: aspectos éticos, históricos e legais no Brasil. Evidência - Ciência e Biotecnologia, v. 19, n. 2, 2019. Disponível <https://portalperiodicos.unoesc.edu.br/evidencia/article/view/20964>. doi: https://doi.org/10.18593/eba.v19i2.20964.

ESPÍRITO SANTO. Lei № 11.325, de 12 de julho de 2021. Proíbe a utilização de animais para desenvolvimento, experimentos e testes de produtos cosméticos, de higiene pessoal, perfumes, limpeza e seus componentes no âmbito do Estado do Espírito Santo e dá outras providências. Diário Oficial do Estado do Espírito Santo, 2021. Disponível em: <https://www.legisweb.com.br/legislacao/?id=417155>.

FURLAN, A.L.D.; FISHER, M.L. Métodos alternativos ao uso de animais como recurso didático: um novo paradigma bioético para o ensino da zoologia. Educação em Revista, $\quad$ v. 36, $2020 . \quad$ Disponível em: <https://www.scielo.br/j/edur/a/SvzX4qmqNKh7JFSZppbz6WJ/?lang=pt>. $\quad$ doi: 10.1590/0102-4698230590.

ICTB - Instituto de Ciência e Tecnologia em Biomodelos. Pesquisa e ensino no ICTB. Consulta institucional, 2021. Disponível em: <https://www.ictb.fiocruz.br/>.

MATO GROSSO DO SUL. Lei № 4.538, de 03 de junho de 2014. Proíbe a utilização de animais para desenvolvimento, experimento e teste de produtos cosméticos e de higiene pessoal, perfumes e seus componentes e dá outras providências. Diário Oficial do Estado do Mato grosso do Sul, 2014. Disponível em: $<$ https://www.legisweb.com.br/legislacao/?id=271023>.

MINAS GERAIS. Lei № 23.050, de 25 de julho de 2018. Proíbe a utilização, no Estado, de animais para desenvolvimento, experimento e teste de perfumes e produtos cosméticos e de higiene pessoal e seus componentes. Diário Oficial do Estado de Minas Gerais, 2018. Disponível em: <https://www.legisweb.com.br/legislacao/?id=365473>.

MORETTO, L.D.; STEPHANO, M.A. Métodos alternativos ao uso de animais em pesquisa reconhecidos no Brasil. São Paulo: Limay, 2019. 732 p. ISBN: 978-8566138-01-06. 
OECD - Organization for Economic Cooperation and Development. Test guidelines programme for the testing of chemicals. 2021. Disponível em: <https://www.oecdilibrary.org/environment/oecd-guidelines-for-the-testing-of-chemicals-section-4health-effects_20745788?_ga=2.164379579.545124248.1629039219613920434.1629039219>.

PARÁ. Lei № 8.361, de 11 de maio de 2016. Proíbe a utilização de animais para desenvolvimento, experimento e teste de produtos cosméticos e de higiene pessoal, perfumes e seus componentes. Diário Oficial do Estado do Pará, 2016. Disponível em: <https://www.legisweb.com.br/legislacao/?id=320483>.

PARANÁ. Lei № 18.668, de 22 de dezembro de 2015. Proíbe a utilização de animais para desenvolvimento de experimentos e testes de produtos cosméticos, de higiene pessoal, perfumes, e seus componentes. Diário Oficial do Estado do Paraná, 2015. Disponível em: <https://www.legisweb.com.br/legislacao/?id=314531>.

PRESGRAVE, O.A.F. The need for the establishment of a Brazilian Centre for the validation of alternative methods (BraCVAM). Alternatives to Laboratory Animals, v. $36, \quad$ p. $705-708, \quad 2008 . \quad$ Disponível em: <https://pubmed.ncbi.nlm.nih.gov/19154096/>. doi: 10.1177/026119290803600613.

RIO DE JANEIRO. Lei № 7.814, de 15 de dezembro de 2017. Proíbe a utilização de animais para desenvolvimento, experimentos e testes de produtos cosméticos, higiene pessoal, perfumes, limpeza e seus componentes, no âmbito do Estado do Rio de Janeiro, sem prejuízo de proibições e sanções previstas em outros dispositivos legais: Municipal, Estadual ou Federal, e dá outras providências. Diário Oficial do Estado do Rio de Janeiro, 2017. Disponível em: <http://alerjln1.alerj.rj.gov.br/CONTLEI.NSF/c8aa0900025feef6032564ec0060dfff/004 d830341147e03832581fb005bfbf9?OpenDocument>.

RUSSEL, W. M. S.; BURCH, R. L. The Principles of Humane Experimental Technique. Universities Federation for Animal Welfare (UFAW) - Special Edition. 1992. Disponível em: <https://altweb.jhsph.edu/pubs/books/humane_exp/het-toc>.

SANTA CATARINA. Lei № 18.009, de 6 de outubro de 2020. Veda a utilização de animais no desenvolvimento, experimento e testes de produtos de higiene pessoal, cosméticos e perfumes. Diário Oficial do Estado de Santa Catarina, 2020. Disponível em: <https://leisestaduais.com.br/sc/lei-ordinaria-n-18009-2020-santa-catarina-vedaa-utilizacao-de-animais-no-desenvolvimento-experimento-e-testes-de-produtos-dehigiene-pessoal-cosmeticos-e-perfumes>.

SÃO PAULO. Lei № 15.316, de 23 de janeiro de 2014. Proíbe a utilização de animais para desenvolvimento, experimento e teste de produtos cosméticos e de higiene pessoal, perfumes e seus componentes e dá outras providências. Diário Oficial do Estado de São Paulo, 2014. Disponível em: $<$ https://www.al.sp.gov.br/norma/?id=172282>.

SARAIVA, T.V.; CRUZ, M.P.; CARNEIRO, J.F.; SILVA, W.C.B.; NETO, F.G.M.; et al. Atendimento à Lei Arouca no ensino de farmacologia no curso de Medicina, UFC, 
Sobral. Revista Brasileira de Educação Médica, v. 40, n.1, p. 138-143, 2016. Disponível em: <https://www.scielo.br/j/rbem/a/LYnzmbTqWWvVjBYmZFSMR9D/?lang=pt>. $\quad$ doi: 10.1590/1981-52712015v40n1e01522015.

SCHEID, N.M.J.; KONFLANZ, T.L. Contribuições das tecnologias da informação e da comunicação em metodologias alternativas para o uso de animais não humanos em aulas experimentais em ciências biológicas. Libros Universidad Nacional Abierta y a Distância, p. 162-169, 2016. Disponível em: <https://hemeroteca.unad.edu.co/index.php/book/article/view/2667>.

SILVA, C.C.; OLIVEIRA, C.B.N.; CARNEIRO, P.S.; MARENGO, E.B.; MATTOS, K.A.; et al. Métodos alternativos para detecção de pirogênios em produtos e ambientes sujeitos à Vigilância Sanitária: avanços e perspectivas no Brasil a partir do reconhecimento internacional do Teste de Ativação de Monócitos. Vigilância Sanitária em Debate, v.6, n.1, p. 137-149, 2018. Disponível em: <https://www.redalyc.org/journal/5705/570563069015/>. doi: 10.22239/2317$269 \times .01082$.

SILVA, R.M.; SANTORI, R.T.; MIRANDA, J.C. Experimentação animal e ensino. Revista Saúde e Biologia, v. 11, n. 1, p. 90-100, 2016. Disponível em: <http://68.183.29.147/revista/index.php/sabios/article/view/2032>.

TRÉZ, T.A. Considerações sobre o conceito dos 3Rs e o potencial conflito com novas compreensões do animal experimental. Revista Brasileira de Zoociências, v. $19, \quad$ n. 2 , p. $97-113,2018$. Disponível em: <https://periodicos.ufjf.br/index.php/zoociencias/article/view/24741> doi: 10.34019/2596-3325.2018.v19.24741. 